\title{
Development of QPM Version of DHM117 Maize Hybrid Using Marker Assisted Selection
}

\author{
M. Surender ${ }^{1 *}$, Prashant Shetti ${ }^{1}$, Deepti B. Sagare ${ }^{1}$, Ch.V. Durga Rani ${ }^{1}$, \\ Farzana Jabeen ${ }^{1}$, M.R. Sudarshan ${ }^{2}$ and S. Sokka Reddy ${ }^{1}$ \\ ${ }^{1}$ Institute of Biotechnology, Professor Jayashankar Telangana State Agricultural University, \\ Hyderabad, Telangana-500 030, India \\ ${ }^{2}$ MRC, ARI, Rajendranagar, Hyderabad-500 030, Telangana, India \\ *Corresponding author
}

\section{Keywords}

QPM- Quality protein maize, opqaue 2 ,

Tryptophan,

Recurrent parent genome.

Article Info

Accepted:

26 September 2017

Available Online:

10 October 2017

A B S T R A C T

Development of QPM (Quality Protein Maize) with high lysine and tryptophan is foremost important task in enhancing nutritional quality in maize through breeding programme. Marker assisted selection is the most feasible way of developing QPM hybrids in short time. The present investigation deals with conversion of elite normal maize inbred lines BML6 and BML7 (parental lines of DHM117 hybrid) into QPM lines using marker assisted selection. The nutritional quality of maize is enhanced by introgression of the opaque 2 (o2) gene along with numerous modifiers for kernel hardness. To improve the efficiency of QPM breeding, the utility of three simple sequence repeat (SSR) markers viz. umc1066, phi057 and phil12 were used in selection and introgression of the opaqaue 2 gene. Polymorphism was detected between recipient parents (BML7 and BML6) and donor parent (CML181) with umc1066 SSR marker. Foreground selection was exercised in each generation using opaque 2 specific marker umc1066 while background selection was carried out in $\mathrm{BC}_{1} \mathrm{~F}_{1}$ and $\mathrm{BC}_{2} \mathrm{~F}_{1}$ generations to recover the recurrent parent (RPG) genome using SSR markers distributed across the genome. In $\mathrm{BC}_{2} \mathrm{~F}_{1}$ the recovery of recurrent parent was between 90 to $93 \%$ and the plants with highest recovery were selfed to generate advanced generations $\left(\mathrm{BC}_{2} \mathrm{~F}_{2}\right.$ and $\left.\mathrm{BC}_{2} \mathrm{~F}_{3}\right)$. Kernels were screened for endosperm hardness using light box and kernels showing less than $25 \%$ opacity were selected. Rigorous phenotyping was done for plant characters and tryptophan was estimated using colorimetric method. Tryptophan content varied from $0.76 \%$ to $0.95 \%$ in $\mathrm{BC}_{2} \mathrm{~F}_{3}$ derived population of BML6 and $0.72 \%$ to $0.92 \%$ in $\mathrm{BC}_{2} \mathrm{~F}_{3}$ derived population of BML7. Normal looking converted inbreds (CBML6 and CBML7) with high tryptophan and high yield were used for reconstitution of the QPM version of DHM117 maize hybrid. Two cross combinations CB6-36 $\times$ CB7-28 and CB6-36 $\times$ CB7-59 exhibited relatively higher tryptophan content and on par grain yield per plant compared to check DHM 117 which need to be further tested under multi-location trials prior to commercial exploitation.

\section{Introduction}

Maize (Zea mays L.) is a staple food for millions of people in poor countries around the world and it provides $15 \%$ of the world's protein and $20 \%$ of the world's calories.
This indicates maize's status as a paramount crop in the context of global nutrition. But, maize kernels lack the essential amino acids lysine and tryptophan. Deficiency in these 
amino acids causes some of the fatal diseases like pellagra, kwashiorkor etc. where maize is a staple food and also leads to a condition called wet malnutrition.

Maize mutant opaque2 was discovered by Mertz et al., (1964) with high lysine and tryptophan content than the normal maize kernels. But due to some of its undesirable and pleiotropic effects like soft, chalky kernel and low yields it was ultimately rejected in the market. In order to prevent these undesirable traits of opaque 2 varieties, researchers started to combine the opaque 2 maize with genetic modifiers which improves the vitreousness of the kernels. These modifiers overcome the negative pleotropic effects of the $o 2$ gene (Ortega and Bates, 1983). Genotypes with o2 allele and $o 2$ modifiers with elevated lysine and tryptophan level and without negative effect are termed as quality protein maize (QPM) (Bjarnason and Vasal 1992; Geevers and Lake, 1992).

Three SSR markers viz., phi 057, phil12 and umc1066 are discovered that lie within the opaque 2 gene facilitated study and application of the $o 2$ gene (Mbogori et al., 2006). Breeding for QPM genotypes becomes straight forward with the help of SSRs located within the $o 2$ locus, which are useful for the QPM breeders (Babu et al., 2005; Danson et al., 2006). Marker Assisted Selection (MAS) is an appropriate technology for traits such as high lysine in maize and can be a cost effective procedure for selecting o2 locus in breeding populations (Babu et al., 2005 and Gupta et al., 2009).

MAS in combination with conventional breeding techniques can greatly accelerate the introgression of QPM genotype into normal maize. In India, only one QPM hybrid, Vivek Hybrid-9 was released in 2008 (Gupta et al., 2009) using MAS at Vivekananda Parvatiya Krishi Anusandhan Sansthan (VPKAS),
Almora, Uttaranchal. The present investigation has been taken for conversion of elite maize inbreds BML6 and BML7 into QPM with high lysine and tryptophan using marker assisted breeding and developing QPM version of DHM 117.

\section{Materials and Methods}

BML6 female parent and BML7 male parent of popular single cross hybrid DHM 117 were selected as recipients. QPM inbred line CML181 was selected as donor. opaque2 gene based SSR markers viz.,umc1066, phi057 and phi112 (Table 1) were used to check the polymorphism between donor and recipient parents and umc1066 was used for foreground selection. Flanking markers of opaque2: bnlg1200 and bnlg2160 (Table 1) were used for recombinant selection. Six SSR markers viz, mmc0241, umc1216, phi072, bnlg1633, bmcl382 and phi075 (Table 1) were used as endosperm and amino acid modifiers.

Parental polymorphism survey between BML6, BML7 (recurrent parents) and CML181 (donor parent) was carried out by Krishna et al., 2011 using 752 genomic SSR markers covering all ten chromosomes. We selected 70 distinct polymorphic markers between BML6 and CML181 and 83 polymorphic markers between BML7 and CML181 were used for background selection. These polymorphic genomic SSR markers were used for background selection.

The field experiments were carried out at the Maize Research Centre (MRC). Genotyping and Biochemical analysis were carried out at Institute of Biotechnology, Rajendranagar, Hyderabad.

The schematic diagram for conversion of BML 6 and BML7 is illustrated in figure 1. PCR programme for genotyping was 
standardised (Table 2), the amplified products were resolved on $3.0 \%$ metaphor: agarose $(1: 2)$ gel and the gel was observed under gel documentation system.

Scoring of alleles was carried out for foreground and background selection. Heterozygous alleles were represented by ' $\mathrm{H}$ ' and scored as 0.5. Homozygous recipient allele was represented by ' $A$ ' and scored as 1 while homozygous donor allele was represented by letter ' $B$ ' and scored as 0 . Based on the scoring, data was analysed using Graphical Genotypes (GGT2) software (Van Berloo, 2007). RPG was calculated using the formula given by Kumar et al., 2011

$\operatorname{RPG}(\mathrm{G})=\frac{[\mathrm{A}+1 / 2 \mathrm{H}]}{\mathrm{N}}$

Where,

$\mathrm{A}=$ number of markers showing homozygosity for recurrent parent allele

$\mathrm{H}=$ number of markers showing heterozygous state for the parental alleles

$\mathrm{N}=$ total number of parental polymorphic markers screened.

Light box screening of the kernels was carried out to check the opacity. Tryptophan content of maize kernels was estimated using the method described by Hernandez and Bates (1969).

Phenotypic Data analysis was carried out using Analyses of varience (ANOVA) for opaqueness, tryptophan and lysine in the endosperm and other phenotypic characters under complete randomized design (CRD) using windostat 8.0. Pearson's simple correlation coefficients were calculated using MS office Excel.

\section{Results and Discussion}

\section{Parental polymorphism}

Distinct polymorphism was observed between the recipient lines (BML 6 and BML 7) and QPM donor (CML 181) with opaque2 gene specific SSR marker umc 1066 (Plate 1). phi057and phill2 did not show any polymorphism.

Among six amino acid modifiers screened, bnlg1633 showed distinct polymorphism between BML6 and CML181 and mmc0241 shown distinct polymorphism between BML7 and CML 181.

\section{Marker assisted Backcross Breeding programme}

Generation of $\mathbf{F}_{1}$ and molecular confirmation

Crosses were made between recurrent parents with donor during Kharif 2010 to generate $\mathrm{F}_{1}$ seeds. During Rabi 2010-11 $\mathrm{F}_{1}$ crop was raised and true $\mathrm{F}_{1}$ plants were confirmed using umc1066 (Plate 2). The confirmed $\mathrm{F}_{1}$ plants were backcrossed to respective recurrent parents to obtain $\mathrm{BC}_{1} \mathrm{~F}_{1}$ seeds.

Generation of $\quad \mathrm{BC}_{1} \mathrm{~F}_{1}$, foreground, recombinant, amino acid modifiers and background selection

During Kharif $2011 \mathrm{BC}_{1} \mathrm{~F}_{1}$ population was raised with population size of 200 in each cross and foreground selection was done using umc1066. Plants showing heterozygous for opaque 2 were identified and subjected to recombinant selection using flanking markers: bnlg1200 and bnlg2160 (Plate 3) and also screened for amino acid modifiers: bnlg1633 for $\mathrm{BML6}-\mathrm{BC}_{1} \mathrm{~F}_{1}$ population and mmc0241 for $B M L 7-\mathrm{BC}_{1} \mathrm{~F}_{1}$ population (Plate 4). In BML6- $\mathrm{BC}_{1} \mathrm{~F}_{1}, 43$ plants and 64 plants in 
BML7- $\mathrm{BC}_{1} \mathrm{~F}_{1}$, were single recombinants and heterozygous. These single recombinants were used for further amino acid modifier and background screening.

For background selection in BML6- $\mathrm{BC}_{1} \mathrm{~F}_{1}, 70$ SSRs were screened (Plate 5) and the recovery of the genome of recipient parent was varied from $60.93 \%$ to $83.00 \%$. 15 plants showed recovery in between $80-83 \%$ and the remaining has shown less than $80 \%$ RPG (Table 3). The plants with $>80 \%$ RPG were back crossed with BML6 for generating $\mathrm{BC}_{2} \mathrm{~F}_{1}$ population. Similarly, 83 SSRs were used for background selection in BML7$\mathrm{BC}_{1} \mathrm{~F}_{1}$ and $\mathrm{RPG}$ was found in between $58.24 \%$ to $83.00 \%$. Ninteen plants showed recovery between $80-83 \%$ and remaining plants showed less than $80 \%$ RPG (Table 3). The plants with $>80 \%$ RPG were back crossed with BML7 for generating $\mathrm{BC}_{2} \mathrm{~F}_{1}$ population.

Generation of $\quad \mathrm{BC}_{2} \mathrm{~F}_{1}$, foreground, recombinant, amino acid modifiers and background selection

BML6 and BML7 derived $\mathrm{BC}_{2} \mathrm{~F}_{1}$ seeds were sown during Rabi 2011-12.One hundred and forty one plants of each $\mathrm{BC}_{2} \mathrm{~F}_{1}$ populations were selected for foreground selection. In BML6- $\mathrm{BC}_{2} \mathrm{~F}_{1}$ and BML7- $\mathrm{BC}_{2} \mathrm{~F}_{1}, 60$ and 64 plants respectively were with opaque 2 in heterozygous condition $(\mathrm{O} 2 \mathrm{o} 2)$. Foreground selected $\mathrm{BC}_{2} \mathrm{~F}_{1}$-BML6 plants were screened with flanking marker bnlg2160 (plate 6) and amino acid modifier marker bnlg1633. Foreground selected $\mathrm{BC}_{2} \mathrm{~F}_{1}$-BML7 plants were screened for flanking marker bnlg1200. Double recombinants were not observed in both the $\mathrm{BC}_{2} \mathrm{~F}_{1}$ population. In screening for amino acid modifiers less number of desired allele (donor) and some heterozygous alleles were observed in both $\mathrm{BC}_{2} \mathrm{~F}_{1}$ populations, these heterozygous and donor type allele plants were subjected for background selection.
Background markers which were not recovered in $\mathrm{BC}_{1} \mathrm{~F}_{1}$ generation were used for background screening. Marker data was analyzed and manually scored and recurrent parent genome (RPG) was calculated using GGT2 software. In $\mathrm{BC}_{2} \mathrm{~F}_{1}$ population the recovery of recurrent parent genome ranged between 90-94.5\%. Six (p-36, p-36-1, p-38, p$39, \mathrm{p}-49$ and $\mathrm{p}-52)$ and eight (p-11, p-15, p26, p-28, p-32, p-38, p-59 and p-92) plants from BML6 and BML7 derived populations respectively were identified to generate $\mathrm{BC}_{2} \mathrm{~F}_{2}$ families (Table 4). Kernels with desired characters were screened for hardness under light box.

\section{Generation of $\mathrm{BC}_{2} \mathrm{~F}_{2}$, foreground selection} and tryptophan estimation

$\mathrm{BC}_{2} \mathrm{~F}_{2}$ population was raised from selected kernels showing less than $25 \%$ opacity during Kharif 2012 and foreground selection was done for opaque 2 gene using umc1066. 182 BML6- $\mathrm{BC}_{2} \mathrm{~F}_{2}$ and 207 BML7- $\mathrm{BC}_{2} \mathrm{~F}_{2}$ plants were subjected to foreground selection. 44 and 56 individuals exhibited recessive homozygous loci in BML6- $\mathrm{BC}_{2} \mathrm{~F}_{2}$ and BML7$\mathrm{BC}_{2} \mathrm{~F}_{2}$ populations respectively (Plate 7). Kernels of selected families of $\mathrm{BC}_{2} \mathrm{~F}_{2}$ populations (CB6-36, CB6-39, CB7-11, CB728 and $\mathrm{CB} 7-59)$ with desired characters were screened for hardness under light box (Fig. 2) and tryptophan was estimated using colorimetric method and undesired families were discarded.

\section{Generation of $\mathrm{BC}_{2} \mathrm{~F} 3$, foreground, recombinant and amino acid modifier selection, tryptophan estimation and phenotyping}

$\mathrm{BC}_{2} \mathrm{~F}_{3}$ populations of BML6 and BML7 were generated during Rabi 2012-13. Foreground selection, recombinant selection and amino acid modifiers screening was carried out in $\mathrm{BC}_{2} \mathrm{~F}_{3}$. The plants with all desired characters were forwarded for next generation. 
Agronomic and biochemical traits recorded in the $\mathrm{BC}_{2} \mathrm{~F}_{3}$ trial were viz., days to $50 \%$ tasseling, days to $50 \%$ silking, days to maturity, plant height $(\mathrm{cm})$, ear height $(\mathrm{cm})$, ear girth $(\mathrm{cm})$, ear length $(\mathrm{cm})$, number of kernel rows, number of kernels /row, test weight, grain yield/plant (g) and tryptophan concentration $(\%)$.

Ears showing similar morphology with recurrent parents in terms of texture, colour of the grains, size of the grains, row arrangements and size of the ears were selected for further advancement. Tryptophan content varied from $0.76 \%$ to $0.95 \%$ in BML6- $\mathrm{BC}_{2} \mathrm{~F}_{3}$ and $0.72 \%$ to $0.92 \%$ in BML7$\mathrm{BC}_{2} \mathrm{~F}_{3}$ population respectively (Table 5 ). The selected families with all desired characters were selfed to generate $\mathrm{BC}_{2} \mathrm{~F}_{4}$ seeds during Rabi 2012-13.

The converted BML6 and BML7 were designated as CB6 and CB7 with their pedigree plant numbers. The selected families were used for crossing program for producing QPM hybrid. The stable expression of these traits was confirmed through $\mathrm{BC}_{2} \mathrm{~F}_{3}$ families, which represented the final converted QPM versions of the original recipient lines.

The expression of genetic modifiers may be affected by maternal influence. Endosperm is a triploid tissue; one may expect maternal influence since two doses of modifying alleles are contributed by the maternal parent and only one by the paternal parent. Reciprocal differences in crosses between soft opaque and modified opaque have been reported by Vasal, 1994.

Other factors such as genetic background and kernel texture can also alter phenotypic manifestation of modifying genes. Flint genetic backgrounds generally exhibit a higher frequency of modified kernels (Vasal, 1994). Therefore, direct and reciprocal crosses between selected families of $\mathrm{CB} 6$ and CB7 inbreds were performed to generate $F_{1}$ hybrids. In direct cross, CB6-36, CB6-39 as female parent and CB7-11, CB7-28 and CB759 as male parent and in reciprocal crosses it was vice-versa for producing QPM hybrid.

The six $\mathrm{F}_{1}$ hybrids were evaluated during the Rabi 2013-14 and Kharif 2014 along with five converted inbreds, two normal inbred, one QPM inbred, one standard check hybrid DHM117 and one standard reciprocal check hybrid BML7 X BML6 (Table 6).

\section{Evaluation of QPM version of DHM117 hybrid}

During Rabi 2013-14 and Kharif 2014, all crosses (Table 6) along with 5 parents, 3 check inbreds (BML6, BML7 and CML181) one commercial check, DHM117 and reciprocal cross between BML7 X BML6 were sown in Randomized Block Design (RBD) replicated thrice.

Each entry was sown in a row of five meter length with a spacing of $75 \mathrm{~cm}$ between rows and $20 \mathrm{~cm}$ between the plants. Necessary plant protection measures were taken to protect the crop from pests and diseases as per the Maize Research Center recommendations, so as to raise a healthy crop.

Converted inbreds (CB6, CB7) and QPM hybrid purity assessment was checked in Rabi 2013-14 using gene specific umc1066 SSR marker. All the QPM hybrids possessed recessive allele of opaque2. Phenotypic data was recorded on twelve different characters viz., days to 50 per cent tasseling, days to 50 per cent silking, days to maturity, plant height $(\mathrm{cm})$, ear height $(\mathrm{cm})$, ear length $(\mathrm{cm})$, ear girth $(\mathrm{cm})$, number of kernel rows per ear, number of kernels per row, 100 kernel weights (g), grain yield per plant (g) and tryptophan content $(\%)$ in generated hybrids. 
Table.1 Markers used in the study

\begin{tabular}{|c|c|c|c|}
\hline marker & location & Sequence & Reference \\
\hline \multicolumn{4}{|c|}{ Opaque 2 specific markers } \\
\hline \multirow{2}{*}{ umc1066 } & \multirow{2}{*}{7.01} & F ATGGAGCACGTCATCTCAATGG & \multirow{6}{*}{ www.agron.missouri.edu } \\
\hline & & R AGCAGCAGCAACGTCTATGACACT & \\
\hline \multirow{2}{*}{ phi057 } & \multirow{2}{*}{7.01} & F CTCATCAGTGCCGTCGTCCAT & \\
\hline & & R CAGTCGCAAGAAACCGTTGCC & \\
\hline \multirow{2}{*}{ phil12 } & \multirow{2}{*}{7.01} & F TGCCCTGCAGGTTCACATTGAGT & \\
\hline & & R AGGAGTACGCTTGGATGCTCTTC & \\
\hline \multicolumn{4}{|c|}{ Flanking markers } \\
\hline \multirow{2}{*}{ bnlg1200 } & \multirow{2}{*}{7.01} & F CGTCCTCGTTGTTATTCCGT & \multirow{4}{*}{ Babu et al., 2004} \\
\hline & & R GTTCCCTCTCCCTCCCTC & \\
\hline \multirow{2}{*}{ bnlg2160 } & \multirow{2}{*}{ 7.01-02 } & F GAAGCAACCCATTTTCATCC & \\
\hline & & R AGATTGGATTCCTGCCTCCT & \\
\hline \multicolumn{4}{|c|}{ Amino acid modifiers } \\
\hline \multirow{2}{*}{ bnlg1633 } & \multirow{2}{*}{2.07} & F GTACCTCCAGGTTTACGCCA & \multirow{8}{*}{$\begin{array}{c}\text { Wu et al., } 2002 \\
\text { Wang and Larkins } 2001\end{array}$} \\
\hline & & R TCAACTTCTCATGCACCCAT & \\
\hline \multirow{2}{*}{ bnlg2136 } & \multirow{2}{*}{3.04} & F TGC TCC TTC TCG AGC ACC & \\
\hline & & R ATG GAC GTA CGG CAG ACT CT & \\
\hline \multirow{2}{*}{ phi072 } & \multirow{2}{*}{4.01} & F ACCGTGCATGATTAATTTCTCCAGCCTT & \\
\hline & & R GACAGCGCGCAAATGGATTGAACT & \\
\hline \multirow{2}{*}{ bnlg1382 } & \multirow{2}{*}{5.01} & F TTTTCTTTCAAAAATATTCAGAAGC & \\
\hline & & R GCAGGATTTCATCGGTTGTT & \\
\hline \multirow{2}{*}{ phil19 } & \multirow{2}{*}{8.02} & F GGG CTC CAG TTT TCA GTC ATT GG & \multirow{2}{*}{$\mathrm{Wu}$ et al., 2002} \\
\hline & & R ATC TTT CGT GCG GAG GAA TGG TCA & \\
\hline \multirow{2}{*}{ phil15 } & \multirow{2}{*}{ 8.03-8.04 } & F GCT CCG TGT TTC GCC TGA A & \multirow{2}{*}{$\mathrm{Wu}$ et al., 2002} \\
\hline & & R ACC ATC ACC TGA ATC CAT CAC A & \\
\hline hnlal655 & 1003 & F ATT AAA ATC TTG CTG ATG GCG & Wul et al 2002 \\
\hline Dengios & $10.0 \mathrm{~J}$ & R TTC TGT TCC CGC CTG TAC TT & wu ct al., 2002 \\
\hline & & Endosperm modifiers & \\
\hline umclold & 604 & F GAA AGT CGA TCG AGA GAC CCT G & Holing et al 2008 \\
\hline Йтा1014 & 0.04 & R CCC TCT CTT CAC CCC TTC CTT & Honng el al., 2000 \\
\hline umc1216 & 702 & F TTGGTTGTTGGCTCCATATTCA & Danson etal 200 \\
\hline पाme 1210 & 1.02 & R GTTATATGCCCGTGCATTGCTA & Dansont et al., 2000 \\
\hline umclo36 & 702 & F CTG CTG CTC AAG GAG ATG GAG A & Holing et al 2011 \\
\hline Umc 1050 & 1.02 & R GAC ACA CAT GCA CGA GCA GAC T & \\
\hline
\end{tabular}

Table.2 PCR programming for three SSR markers used in the present study

\begin{tabular}{|c|c|c|}
\hline $\begin{array}{l}\text { Steps follwed in } \\
\text { Thermal cycler }\end{array}$ & $\begin{array}{l}\text { Temperature in degree } \\
\text { celcius for one cycle }\end{array}$ & $\begin{array}{c}\text { Time } \\
\text { for one cycle }\end{array}$ \\
\hline Step 1 & $94^{\circ} \mathrm{C}$ & 5 minutes \\
\hline Step 2 & $94^{\circ} \mathrm{C}$ & 0.45 minute \\
\hline Step 3 & $56^{\circ} \mathrm{C}$ & $0.45 \mathrm{~min}$ \\
\hline Step 4 & $72^{\circ} \mathrm{C}$ & 1minute \\
\hline $\begin{array}{l}\text { Aabove } 2-4 \text { steps are } \\
\text { repeated for } 35 \text { cycles } \\
\text { Step } 5\end{array}$ & & \\
\hline Step 6 & $\begin{array}{l}72^{\circ} \mathrm{C} \\
\text { hold at } 4^{\circ} \mathrm{C} \text { until ready to } \\
\text { load onto gel }\end{array}$ & 10 minutes \\
\hline
\end{tabular}


Table.3

\begin{tabular}{|c|c|c|c|c|c|c|c|c|c|c|c|c|}
\hline \multirow[t]{2}{*}{ Population } & \multirow[t]{2}{*}{$\begin{array}{l}\text { Populatio } \\
\text { n size }\end{array}$} & \multicolumn{3}{|c|}{$\begin{array}{l}\text { Foreground } \\
\text { selection }\end{array}$} & \multicolumn{3}{|c|}{$\begin{array}{l}\text { Recombinant } \\
\text { selection }\end{array}$} & \multirow{2}{*}{$\begin{array}{c}\text { No. of } \\
\text { polymorphic } \\
\text { background } \\
\text { markers } \\
\text { screened } \\
\end{array}$} & \multirow[t]{2}{*}{$\begin{array}{l}\text { Range of RPG } \\
\text { rate }(\%)\end{array}$} & \multirow{2}{*}{$\begin{array}{c}\text { Mean } \\
\text { RPG } \\
\text { rate }(\%)\end{array}$} & \multirow[t]{2}{*}{$\begin{array}{c}\text { Individuals with } \\
\text { highest RPG }\end{array}$} & \multirow{2}{*}{$\begin{array}{c}\text { No. of non- } \\
\text { recovered } \\
\text { background } \\
\text { markers }\end{array}$} \\
\hline & & $\mathrm{D}$ & $\mathrm{H}$ & $\mathrm{R}$ & $\mathrm{D}$ & $\mathrm{H}$ & $\mathrm{R}$ & & & & & \\
\hline $\begin{array}{l}\text { BML6- } \\
\text { BC }_{1} F_{1}\end{array}$ & 200 & 0 & 98 & 102 & 55 & 23 & 20 & 70 & $60.93-83.00$ & 78.52 & 15 & 16 \\
\hline $\begin{array}{l}\text { BML7- } \\
\mathrm{BC}_{1} \mathrm{~F}_{1}\end{array}$ & 200 & 0 & 103 & 97 & 40 & 36 & 27 & 83 & $58.24-83.00$ & 76.20 & 19 & 17 \\
\hline
\end{tabular}

Table.4

\begin{tabular}{|c|c|c|c|c|c|c|c|c|c|c|c|c|}
\hline \multirow[t]{2}{*}{ Population } & \multirow[t]{2}{*}{$\begin{array}{l}\text { Population } \\
\text { size }\end{array}$} & \multicolumn{3}{|c|}{$\begin{array}{c}\text { Foreground } \\
\text { selection }\end{array}$} & \multicolumn{3}{|c|}{$\begin{array}{c}\text { Recombinant } \\
\text { selection }\end{array}$} & \multirow{2}{*}{$\begin{array}{c}\text { No. of } \\
\text { polymorphic } \\
\text { background } \\
\text { markers } \\
\text { screened }\end{array}$} & \multirow[t]{2}{*}{$\begin{array}{c}\text { Range of RPG } \\
\text { rate }(\%)\end{array}$} & \multirow{2}{*}{$\begin{array}{c}\text { Mean } \\
\text { RPG } \\
\text { rate }(\%)\end{array}$} & \multirow[t]{2}{*}{$\begin{array}{c}\text { Individuals with } \\
\text { highest RPG }\end{array}$} & \multirow{2}{*}{$\begin{array}{c}\text { No. of non- } \\
\text { recovered } \\
\text { background } \\
\text { markers }\end{array}$} \\
\hline & & $\mathrm{D}$ & $\mathrm{H}$ & $\mathrm{R}$ & $\mathrm{D}$ & $\mathrm{H}$ & $\mathrm{R}$ & & & & & \\
\hline $\begin{array}{l}\text { BML6- } \\
\mathrm{BC}_{2} \mathrm{~F}_{1}\end{array}$ & 140 & 0 & 60 & 80 & 20 & 27 & 3 & 16 & $87-93.5$ & 89.32 & 6 & 8 \\
\hline $\begin{array}{l}\text { BML7- } \\
\text { BC }_{2} F_{1}\end{array}$ & 130 & 0 & 64 & 76 & 23 & 36 & 5 & 17 & $85-94.5$ & 90.56 & 8 & 7 \\
\hline
\end{tabular}

Table.5 Tryptophan content of the converted inbreds, normal inbreds and donor

\begin{tabular}{|c|c|c|c|c|c|}
\hline S. No. & BML6 - $\mathrm{BC}_{2} \mathrm{~F}_{3}$ & Tryptophan content $(\%)$ & S. No. & $\mathrm{BML7}-\mathrm{BC}_{2} \mathrm{~F}_{3}$ & Tryptophan content (\%) \\
\hline 1 & $36-1$ & 0.95 & 1 & 11 & 0.81 \\
\hline 2 & $36-2$ & 0.86 & 2 & 15 & 0.92 \\
\hline 3 & 38 & 0.79 & 3 & 26 & 0.74 \\
\hline 4 & 39 & 0.81 & 4 & 28 & 0.87 \\
\hline 5 & 49 & 0.76 & 5 & 32 & 0.79 \\
\hline 6 & 52 & 0.83 & 6 & 38 & 0.81 \\
\hline Control & BML6 & 0.39 & 7 & 59 & 0.86 \\
\hline Control & BML7 & 0.42 & 8 & 92 & 0.82 \\
\hline Donor & CML181 & 0.93 & & & \\
\hline
\end{tabular}

Table.6 Crosses attempted for making direct and reciprocal hybrids

\begin{tabular}{|c|c|c|c|}
\hline S.No & Direct & S.No & Reciprocal \\
\hline 1 & CB6-36 X CB7-28 & 1 & CB7-11 XCB6-36 \\
\hline 2 & CB6-36 X CB7-28 & 2 & CB7-11 XCB6-39 \\
\hline 3 & CB6-36 X CB7-59 & 3 & CB7-28 X CB6-36 \\
\hline 4 & CB6-39 X CB7-11 & 4 & CB7-28 X CB6-39 \\
\hline 5 & CB-39 X CB7-28 & 5 & CB7-59 XCB6-36 \\
\hline 6 & CB-39 X CB7-59 & 6 & CB7-59 XCB6-39 \\
\hline Check & DHM117 (BML6 X BML7) & Check & BML7 X BML6 \\
\hline
\end{tabular}


Table.7 Analysis of variance for randomized block design for yield and yield attributing characters in maize during Rabi 2013-14

\begin{tabular}{|c|c|c|c|}
\hline \multirow[t]{2}{*}{ Character } & \multicolumn{3}{|c|}{ Mean sum of square } \\
\hline & $\begin{array}{l}\text { Replication } \\
(\mathrm{d} . \mathrm{f}=2)\end{array}$ & $\begin{array}{l}\text { Genotypes } \\
(\text { d.f }=21)\end{array}$ & $\begin{array}{l}\text { error } \\
(\mathrm{d} . \mathrm{f}=42)\end{array}$ \\
\hline Days to $50 \%$ tasseling & 1.84 & $31.56 * * *$ & 1.41 \\
\hline Days to $50 \%$ silking & 0.173 & $32.29 * * *$ & 1.09 \\
\hline Days to maturity & $2.695 *$ & $16.94 * * *$ & 0.77 \\
\hline Plant height $(\mathrm{cm})$ & 3.725 & $2181.64 * * *$ & 2.83 \\
\hline Ear height $(\mathrm{cm})$ & $7.501 * *$ & $737.67 * *$ & 1.03 \\
\hline Ear length $(\mathrm{cm})$ & 0.174 & $19.19 * * *$ & 0.08 \\
\hline Ear girth $(\mathrm{cm})$ & $0.143 * *$ & $10.60 * * *$ & 0.03 \\
\hline $\begin{array}{l}\text { Number of kernel rows } \\
\text { per ear }\end{array}$ & 0.002 & $2.30 * * *$ & 0.04 \\
\hline $\begin{array}{l}\text { Number of kernels per } \\
\text { row }\end{array}$ & 0.335 & $193.06 * * *$ & 0.34 \\
\hline 100 kernel weight $(\mathrm{g})$ & 1.832 & $183.56 * * *$ & 1.74 \\
\hline $\begin{array}{l}\text { Grain yield per plant } \\
(\mathrm{g})\end{array}$ & 23.214 & $5719.36^{* * *}$ & 3.41 \\
\hline Tryptophan content (\%) & 0.000 & $0.066 * *$ & 0.00 \\
\hline
\end{tabular}

*significant at $5 \%$ level; ** significant at $1 \%$ level

Table.8 Analysis of variance for randomized block design for yield and yield attributing characters in maize during Kharif 2014

\begin{tabular}{|l|l|l|l|}
\hline \multirow{2}{*}{ Character } & \multicolumn{3}{|c|}{ Mean sum of square } \\
\cline { 2 - 4 } & $\begin{array}{l}\text { Replication } \\
(\mathrm{d} . \mathrm{f}=2)\end{array}$ & $\begin{array}{c}\text { Genotypes } \\
(\mathrm{d} . \mathrm{f}=21)\end{array}$ & $\begin{array}{c}\text { error } \\
(\mathrm{d} . \mathrm{f}=42)\end{array}$ \\
\hline Days to 50\% tasseling & 0.154 & $27.80 * * *$ & 0.62 \\
\hline Days to 50\% silking & 0.466 & $25.93 * * *$ & 0.58 \\
\hline Days to maturity & $9.577 * *$ & $11.43 * * *$ & 1.27 \\
\hline Plant height(cm) & 339.581 & $2923.94 * * *$ & 351.49 \\
\hline Ear height $(\mathrm{cm})$ & 0.009 & $713.49 * * *$ & 2.10 \\
\hline Ear length $(\mathrm{cm})$ & 0.063 & $18.68 * * *$ & 0.033 \\
\hline Ear girth $(\mathrm{cm})$ & 0.031 & $9.84 * * *$ & 0.024 \\
\hline $\begin{array}{l}\text { Number of kernel rows per } \\
\text { ear }\end{array}$ & 0.016 & $2.18 * * *$ & 0.025 \\
\hline Number of kernels per row & 0.329 & $205.48^{* * *}$ & 0.48 \\
\hline 100 kernel weight (g) & 0.44 & $178.39 * * *$ & 0.14 \\
\hline Grain yield per plant $(\mathrm{g})$ & $57.041 * * *$ & $5604.50 * * *$ & 3.16 \\
\hline Tryptophan content $(\%)$ & $0.001 * * *$ & $0.07 * * *$ & 0.00 \\
\hline
\end{tabular}

*significant at $5 \%$ level; ** significant at $1 \%$ level 
Fig.1 Schematic diagram for conversion of BML 6 and BML 7 inbreds to QPM version and reconstitution of QPM hybrid

BML 6 X CML 181

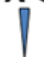

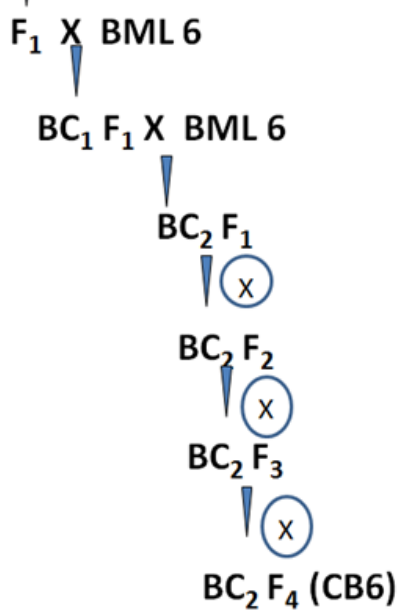

BML 7 X CML 181
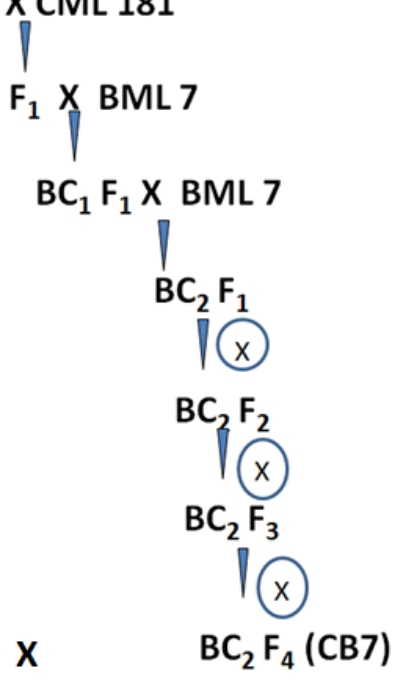

$\mathrm{X} \quad \mathrm{BC}_{2} \mathrm{~F}_{4}(\mathrm{CB} 7)$
Foreground selection

Foreground \& parental polymorphism survey

Foreground \& Background Selection

Foreground \&

Background Selection

Foreground selection and Phenotyping

Plant phenotyping and tryptophan estimation Inbred (CB6\&CB7) evaluation and attempting crosses

\section{QPM $F_{1}$ Hybrid evaluation}

\section{7 \\ Seed production}

Fig.2 Light box screening for endosperm modification of BML6- $\mathrm{BC}_{2} \mathrm{~F}_{2}$ and $\mathrm{BML} 7-\mathrm{BC}_{2} \mathrm{~F}_{2}$ individuals

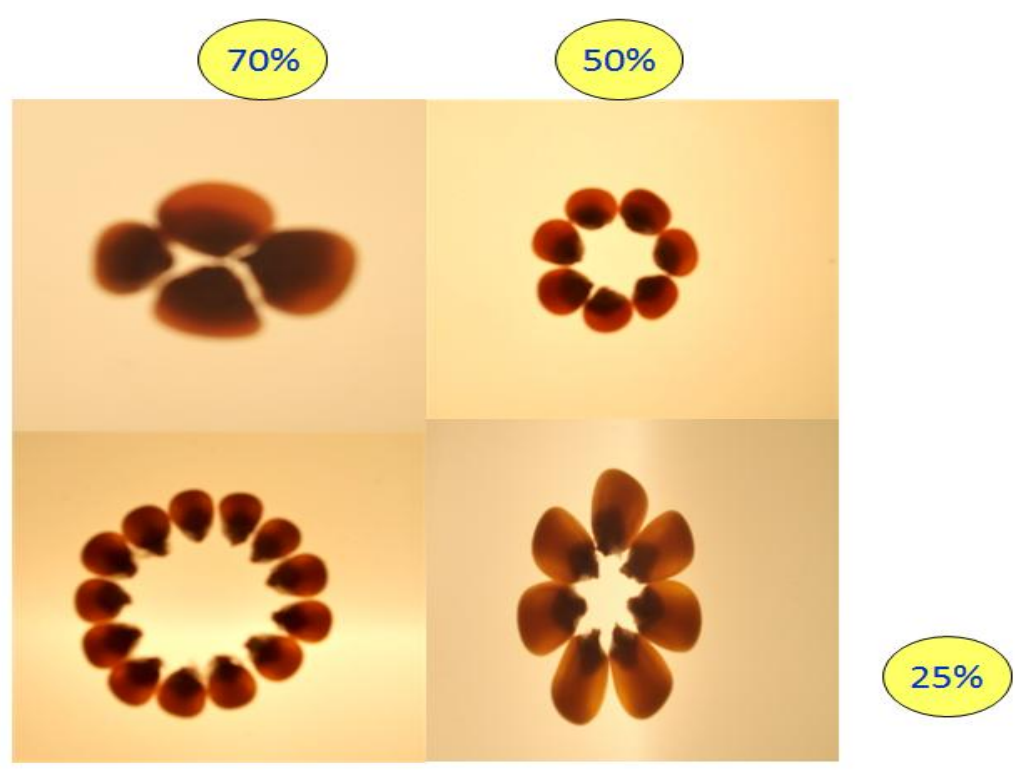


Plate.1 Parental polymorphism for umc 1066

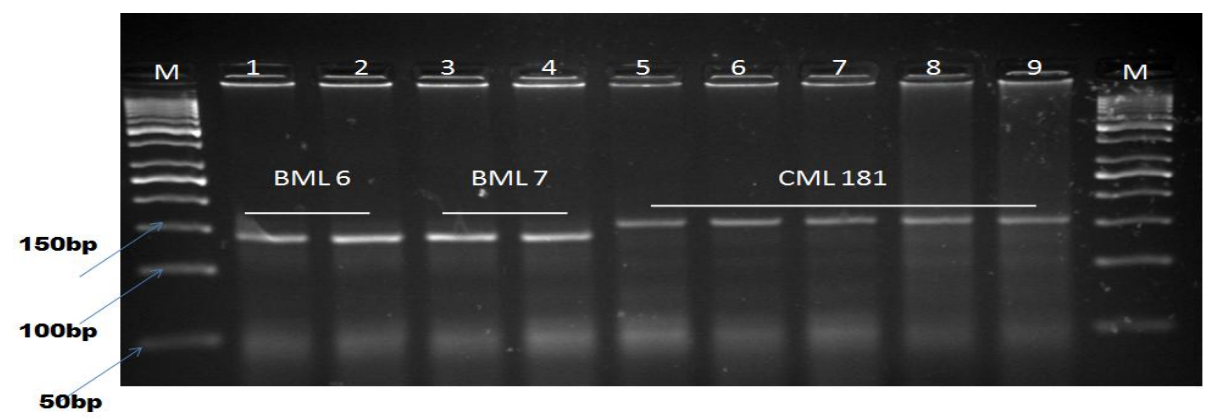

M: 50 bp ladder; 1-2: BML6; 3-4: BML7; 5-9: CML181

Plate.2 $\mathrm{F}_{1}$ (BML7 X CML181) confirmation by umc1066 marker

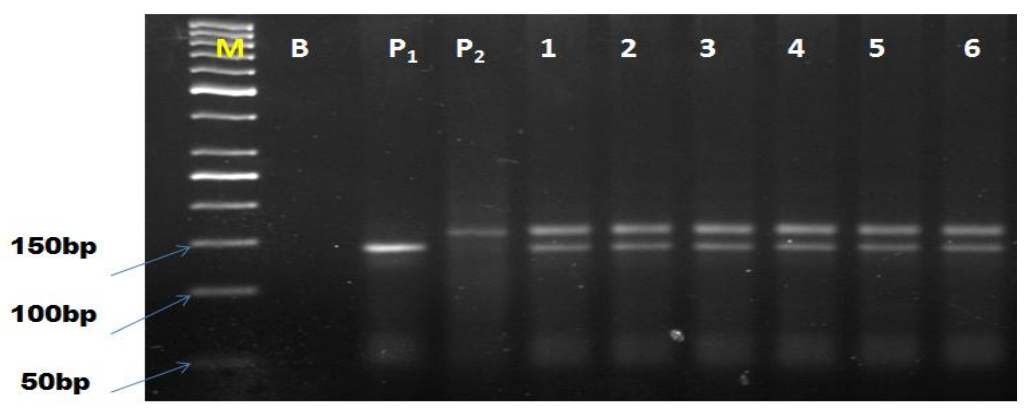

M: 50bp ladder; B: Blank; $\mathrm{P}_{1}$ : BML 7; $\mathrm{P}_{2}$ :CML181; 1-6 $\mathrm{F}_{1}$ individuals

Plate.3 Recombinant screening of BML6- $\mathrm{BC}_{1} \mathrm{~F}_{1}$ population with bnlg2160

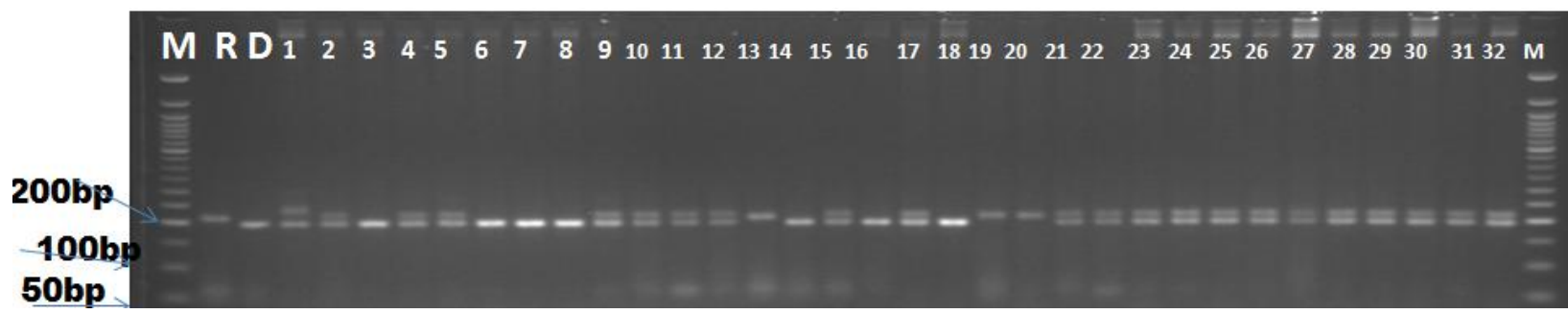

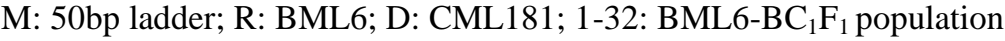

Plate.4 Screening of $\mathrm{BML7}-\mathrm{BC}_{1} \mathrm{~F}_{1}$ population with amino acid modifier $m m c 0241$

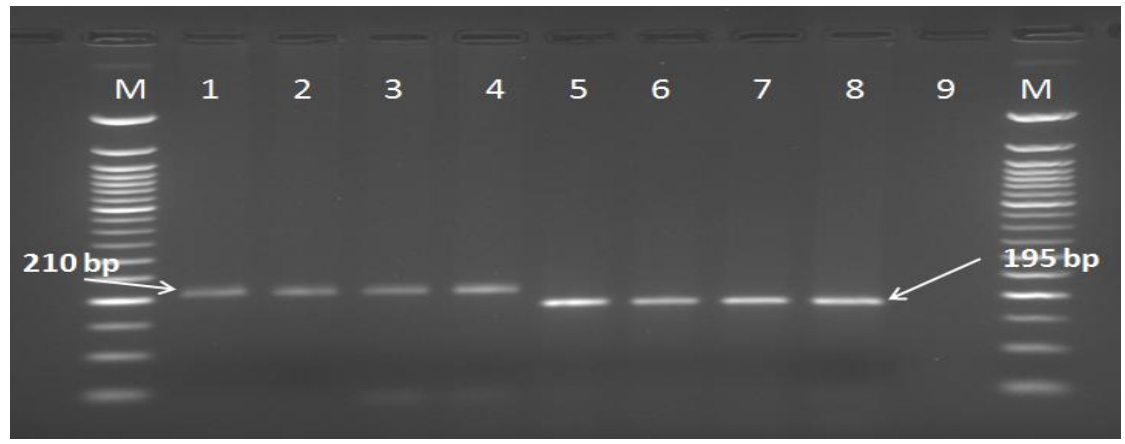

M: 50 bp ladder; B: Blank; 1-4 : BML 7; 5-8 : CML 181. 
Plate.5 Background screening of BML6- $\mathrm{BC}_{1} \mathrm{~F}_{1}$ population with bmc 1655

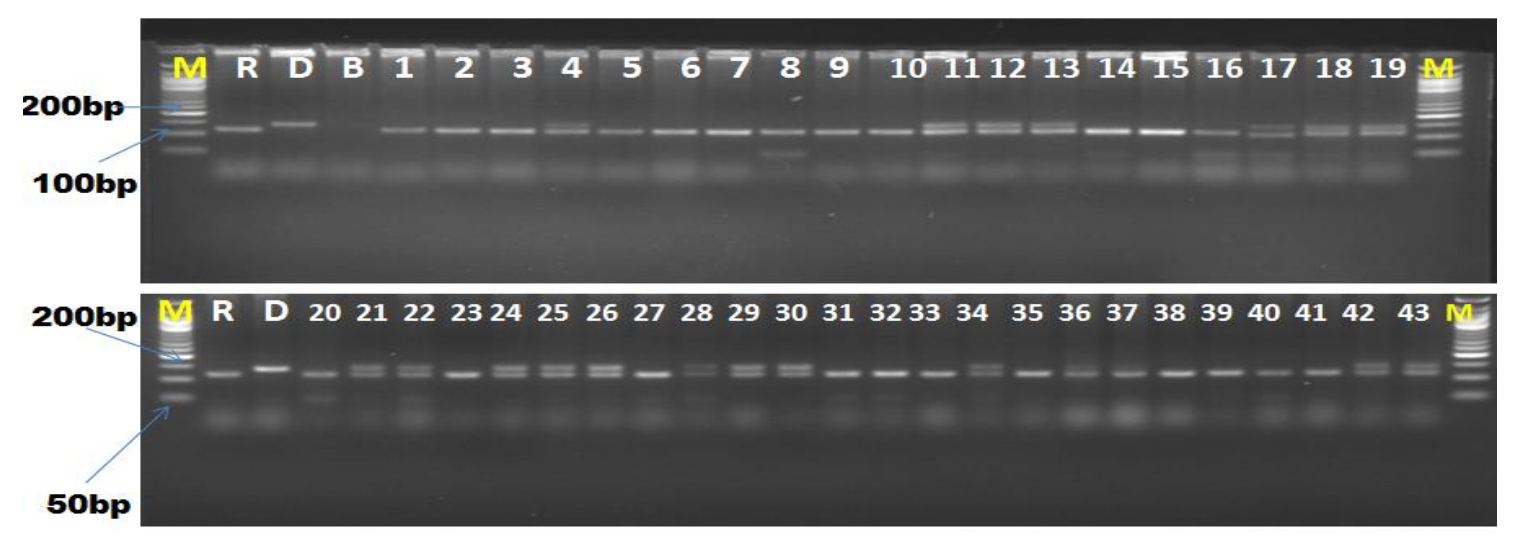

Plate.6 Recombinant screening of BML6- $\mathrm{BC}_{2} \mathrm{~F}_{1}$ population with bnlg2160

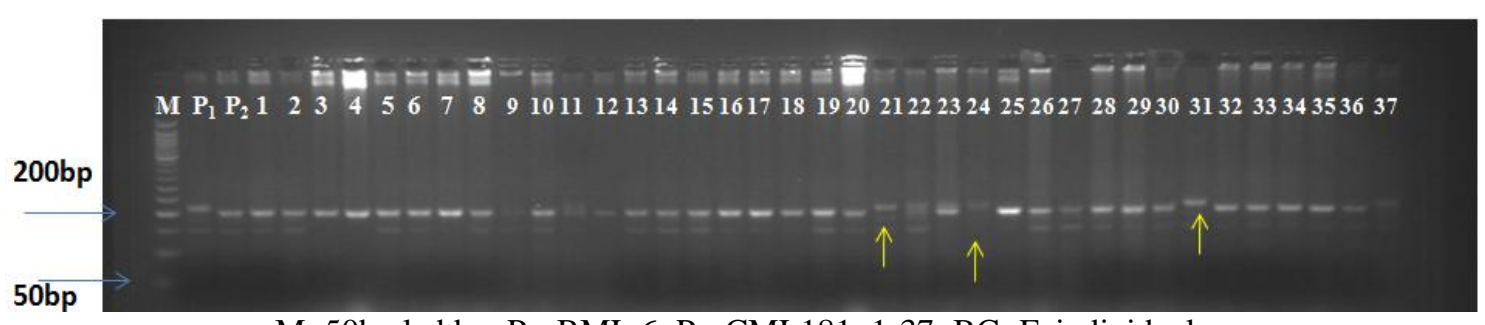

M: 50bp ladder; $\mathrm{P}_{1}$ : $\mathrm{BML}$ 6; $\mathrm{P}_{2}$ : CML181; 1-37: $\mathrm{BC}_{2} \mathrm{~F}_{1}$ individuals

Plate.7 Foreground screening of $\mathrm{BML7}-\mathrm{BC}_{2} \mathrm{~F}_{2}$ population with umc1066

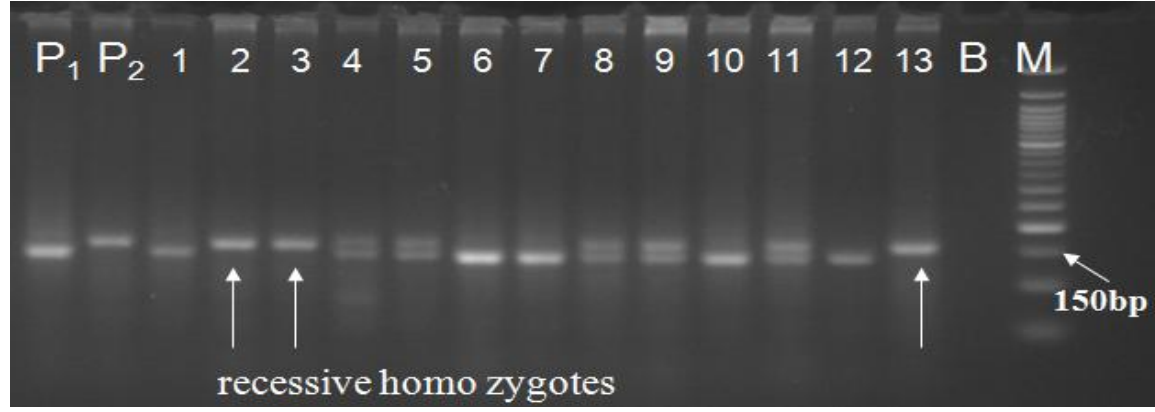

M: 50bp ladder; $\mathrm{P}_{1}$ : BML7; $\mathrm{P}_{2}$ : CML181; 1-13: $\mathrm{BML7-}-\mathrm{BC}_{2} \mathrm{~F}_{2}$ individuals

\section{Hybrid evaluation}

The data was recorded on twelve different characters viz., days to 50 per cent tasseling, days to 50 per cent silking, days to maturity, plant height $(\mathrm{cm})$, ear height $(\mathrm{cm})$, ear length $(\mathrm{cm})$, ear girth $(\mathrm{cm})$, number of kernel rows per ear, number of kernels per row, 100 kernel weights $(\mathrm{g})$, grain yield per plant $(\mathrm{g})$ and tryptophan content (\%).(Unpublished).

\section{Analysis of variance for Rabi 2013-14}

The analysis of variance of the parents and the hybrids for yield and yield attributing characters are presented in Table 7. The mean sum of squares for genotypes (parents and hybrids) was highly significant for all the traits studied. While, the mean sum of squares for replications was found to be nonsignificant for all the traits except for days to 
maturity, ear height and ear girth. There by indicating the existence of sufficient variability in the material studied (Fisher and Yates, 1967).

\section{Analysis of variance for Kharif 2014}

The analysis of variance of the parents and the hybrids for yield and yield attributing characters are presented in Table 8.

The mean sum of squares for genotypes (parents and hybrids) was highly significant for all the traits studied.

While, the mean sum of squares for replications was found to be non-significant for all the traits except for days to maturity, grain yield per plant and tryptophan content. There by indicating the existence of sufficient variability in the material studied (Fisher and Yates, 1967).

\section{Grain yield per plant (g)}

Among the QPM hybrids, maximum grain yield per plant was recorded by CB7-59 X CB6-36 (171.34 g) and was significantly superior when compared to the remaining hybrids for this trait. When compared with the standard check DHM 117, six hybrids were on par for grain yield per plant, but remaining six hybrids were recorded significantly superior mean values over DHM117(Unpublished).

\section{Tryptophan content (\%)}

Among the hybrids, maximum kernel tryptophan content was recorded by CB6-36 X CB7-11 (0.88) and was significantly superior when compared to the remaining QPM hybrids for this trait. When compared with the standard check DHM 117, all hybrids were recorded significantly superior mean values over DHM 117.
Marker assisted selection for opaque 2 gene

In this study, distinct polymorphism could be observed between the normal and QPM inbred lines with two based SSR markers namely umc1066 and phi112. The QPM donor CML181 is an excellent Mexican donor according to Vivek et al., 2007. The SSR marker umc1066, which is located within the middle of the opaque 2 gene. This umc1066 marker shown an excellent allelic polymorphism between donor inbred and recipient inbreds. This marker enables their potential utility in our marker assisted selection study. umc1066 marker is capable of discriminating homozygotes from heterozygotes and is a good co-dominant marker similar type of results was reported in the earlier studies(Babu et al., 2005; Ignjatovic-Micic et al., 2009 and Singh et al., 2012). Identification of heterozygotes and dominant homozygotes in the early stage prior to pollination in the backcross program.

Based on the earlier studies by Lee (1995), Riabut et al., (2002), Babu et al., 2005 and Gupta et al., 2009, fore ground selection is for discriminating recurrent allele, donor allele and heterozygote, recombinant selection for reducing linkage drag with the help of closest flanking markers and background selection is for selecting high recurrent parent genome proportion in the population. Flanking marker analysis was done for reduction of the proportion on the carrier chromosome around the target al.,lele (linkage drag) and reduction of the donor genome on the non-carrier chromosomes to the maximum extent. In this study, we used closest flanking SSR markers near to opaque 2 gene bnlg1200 and bnlg 2100. It was already reported in the earlier study (Babu et al., 2005)

Reduction of the proportion of the donor genome on the carrier chromosomes to the 
maximum extent. In this study, we used flanking markers bnlg1200 and bnlg2100 based on the earlier studies by Babu et al., 2005 and Ribaut et al., 2002, it could be generalized that where a target gene is introduced for the first time presumably from either wild or unadapted germ plasm, flanking markers as close as $2 \mathrm{cM}$ is considered the ideal option, while in the transfer of the same target gene in subsequent phases from elite into elite line. In this study, although the donor QPM inbred line CML 181(F) is an elite line with proven combining ability, with orange kernels with $0.96 \%$ tryptophan content and belongs to late maturity group.

Background selection for recovery of maximum recurrent genome percentage (RPG): The objective of the whole genome selection is to recover rapidly maximum proportion of recurrent parent genome at nontarget loci through background polymorphic markers distributed throughout the genome (Young and Tanksley 1989, Babu et al., 2005 and Gupta et al., 2015).

In the present investigation we fallowed a two generation marker based breeding program in which whole genome background selection at non-target loci was applied in the $\mathrm{BC}_{1}$ and $\mathrm{BC}_{2}$ generations. In our study a total of 153 polymorphic SSR markers used for background selection and our results was co inside with earlier reports.

Based on the marker aided background analysis, an individual with high proportion of RPG needs to be chosen and forwarded for next generations. We selected the first three individuals with highest proportion of recurrent parent genome for developing further $\mathrm{BC}_{2} \mathrm{~F}_{2}$ families. These could be several modifications to the procedure we have fallowed in this study depending up on the requirements and objectives of each breeding scheme.

\section{Phenotyping}

The opaque 2 allele is recessive and the endosperm modifiers are polygenic with, no reliable molecular markers identified for kernel modification. Some of the SSR markers reported endosperm modifier and amino acid modifier were used in the present study. Phenotypic screening of the individual kernels under transmitted light and selection of kernels that have less than $25 \%$ opaqueness is the most convenient and efficient strategy employed in all the QPM breeding programs (Vasal et al., 1993). We preferred kernels with less than $25 \%$ b opaqueness over $25-50 \%$ and more than $50 \%$ opaqueness due to the semi soft nature of endosperm and susceptibility to store grain pest and ear rots.

The tryptophan analysis of the kernel was carried out with Hernandez and Bates (1969) protocol. Phenotypic selection was exercised in the marker assisted opaque2 homozygous individuals in the three $\mathrm{BC}_{2} \mathrm{~F}_{2}$ families for desirable yield and yield attributing characters such as days to $50 \%$ flowering, plant height, ear height, number of kernel rows, number of kernels per row test weight, tryptophan content etc.

The stable expression of these opaque 2 traits could be confirmed through $\mathrm{BC}_{2} \mathrm{~F}_{3}$ families; which reported the final converted QPM version of CB6 and CB7 showed 46\% superiority with tryptophan concentration of above $0.83 \%$ in endosperm protein. Direct and reciprocal crosses between selected families of CB6 and CB7 inbreds were prformed to generate $F_{1}$ hybrids. In direct cross, CB6-36, CB6-39 as female parent and CB7-11, CB7-28 and CB7-59 as male parent and in reciprocal crosses it was vice-versa for producing QPM hybrid. Two cross combinations CB6-36 × CB7-28 and CB6-36 $\times$ CB7-59 exhibited relatively higher tryptophan content and on par grain yield per 
plant compared to check DHM 117 which need to be further tested under multi-location trials prior to commercial exploitation.

The most important goal of QPM research has been to reduce malnutrition in target countries through direct human consumption, even though the impact as of now, has been great perceived. It is expected that greater impact will accrue out of development and dissemination of improved QPM hybrids worldwide.

The converted QPM inbreds (CBML6 and CBML7) developed in the present study provides an ideal platform for stacking number of nutritionally important traits such as enhanced $\mathrm{Fe}$ and $\mathrm{Zn}$, low phytate (for increased bioavailability of nutrients) and high provitamin A. Considering the pace and the technological developments in genomics and proteomics, molecular breeding will be most leading option in future for stacking nutritionally important traits in maize.

Cross combination CB6-36 X CB7-28 recorded higher yield (170g/plant) which is on par with DHM117 hybrid with increased tryptophan $(0.78 \%)$ content. New QPM inbreds developed (CB6-36, CB6-30, CB639, CB7-11, CB7-28 and CB7-59) are useful for further breeding programs for development of improved QPM hybrids.

We further need to develop high throughput, low cost, easily accessible phenotying/screening tools. Generating awareness among the society and building global and national partners for eliminating malnutrition will be future strengths for biofortified maize. Effective seed production and distribution systems, market strategies, strong partnership among research groups and need based government policies will help in solving the problems of many poor and undernourished people.

\section{Acknowledgements}

The work was supported by grants from Department of Biotechnology, Government of India. First author is thankful to UGC, Govt. of India for providing fellowship to pursue Ph.D. All authors are thankful to PJTSAU for providing facilities to conduct the research.

\section{References}

Bjarnason, M., and Vasal, S. K. 1992. Breeding of quality protein maize (QPM). Plant Breeding Review. 9:181216.

Danson, J., Mbogori, M., Kimani, M., Lagat M., Kuria, A and Diallo, A. 2006. Marker assisted introgression of opaque 2 gene in to herbicide tolerant elite maize inbred lines: African Journal of Biotechnology. 5: 2417-2422.

Fisher, R.A., and Yates, F. 1967. Statistical tables for Biological, Agricultural and Medical Research, Longmen Group Limited. London.

Gevers, H. O., and Lake, J. L. 1992. Development of modified opaque 2 maize, In Quality protein maize. E.T. Mertz (ed.) American Association of Cereal Chemistry. pp. 49.

Gupta, H. S., Agarwal P K and Mahajan V, 2009. Quality protein maize for nutritional security: Rapid development of short duration hybrids through molecular marker assisted breeding. Current Science. 96: 230-237.

Hernandez, H. H., and Bates, L. S. 1969. Amodified method for a rapid tryptophan analysis in maize. Research Bulletin, 13. CIMMYT, Mexico City, Mexico, pp.7.

Ignjatovic, V., Mara Kovic, K., Ristic, D., Mladenovic Drinic, S., Stankovic, S., Jancic, L and Denic, M. 2009. Variability analysis of normal and 
opaque 2 maize inbred lines. Genetika. 41(1) $81-93$.

Krishna, M. S. R., 2012. Marker Assisted Selection for conversion of elite Maize inbreds into HQPM lines with high Lysine and Tryptophan. Ph.D Thesis. Acharya N.G. Ranga Agricultural University, Hyderabad, India.

Kumar, J., Jaiswal, V., Kumar, A., Kumar, N., Mir, R.R., Kumar, S., Dhariwal, R., Tyagi, S., Khandelwal, M., Prabhu, K.V., Prasad, R., Balyan, H.S and Gupta, P.K. 2011.Introgression of a major gene for high grain protein content in some Indian breadwheat cultivars. Field Crops Research. 123: 226-233.

Mbogori, M. N., Kimani, M., Kuria, A., Lagat, M and Danson, J. W. 2006. Optimization of FTA technology for large scale plant DNA isolation for use in marker assisted selection. African Journal of Biotechnology. 5(9): 693696.

Mertz, E. T., Bates, L. S and Nelson, O. E. 1964. Mutant genes that change protein composition and increase lysine content of maize endosperm. Science. 145: 279280.

Ortega, E.I., and Bates, L.S. 1983. Biochemical and agronomic studies of two modified hard-endosperm opaque2 maize (Zea mays L.) populations. Cereal Chemistry. 60: 107-111.

Rajesh, V., Kumar, S.S., Reddy, V.N and Sankar, A.S. 2014. Heterosis studies for grain yield and its component traits in single cross hybrids of maize (Zea mays L.). International Journal of Plant, Animal and Environmental Sciences. 4 (1): 304-306.

Ribaut, J.M., and Hoisington, D. 2002. Stimulation experiments on efficiencies of gene introgression by back crossing. Crop Science. 42: 557-565.

Singh, R., and Ram, L. 2012. DNA Aided Introgression of Opaque-2 Allele for Development of Quality Protein Maize. International Journal of Science and Research, ISSN (Online): 2319-7064.

Van Berloo, R., 2007. Graphical genotypes user manual (GGt2.0).

Vasal, S.K., 1994. High quality protein corn,.In Specialty Corns. A.R. Hallauer (ed.) CRC Press, Boca Raton, Florida. pp 79-121.

Young, N. D., Tanksley, S. D. 1989. RFLP analysis of the size of chromosomal segments retained around Tm-2 locus of tomato during back cross breeding. Theoretical and applied Genetics. 77:353-359.

\section{How to cite this article:}

Surender, M., Prashant Shetti, Deepti B. Sagare, Ch.V. Durga Rani, Farzana Jabeen, M.R. Sudarshan and Sokka Reddy, S. 2017. Development of QPM Version of DHM117 Maize Hybrid Using Marker Assisted Selection. Int.J.Curr.Microbiol.App.Sci. 6(10): 3275-3289. doi: https://doi.org/10.20546/ijcmas.2017.610.384 